\title{
Trivium
}

Revue franco-allemande de sciences humaines et sociales - Deutsch-französische Zeitschrift für Geistesund Sozialwissenschaften

11 | 2012

Gustave Flaubert. A l'Orient du réalisme

\section{Le réalisme de Flaubert}

Introduction

\section{Barbara Vinken}

Traducteur : Claudine Layre

\section{OpenEdition}

\section{Journals}

Édition électronique

URL : http://journals.openedition.org/trivium/4247

DOI : $10.4000 /$ trivium.4247

ISSN : 1963-1820

Éditeur

Les éditions de la Maison des sciences de l'Homme

\section{Référence électronique}

Barbara Vinken, « Le réalisme de Flaubert », Trivium [En ligne], 11 | 2012, mis en ligne le 29 juin 2012 consulté le 22 septembre 2020. URL : http://journals.openedition.org/trivium/4247 ; DOI : https:// doi.org/10.4000/trivium.4247

Ce document a été généré automatiquement le 22 septembre 2020.

\section{c) () $\Theta$}

Les contenus des la revue Trivium sont mis à disposition selon les termes de la Licence Creative Commons Attribution - Pas d'Utilisation Commerciale - Pas de Modification 4.0 International. 


\title{
Le réalisme de Flaubert
}

\author{
Introduction
}

Barbara Vinken

Traduction : Claudine Layre

1 Permettez-moi de commencer par une citation de Flaubert qui surprendra peut-être, y compris ses plus parfaits connaisseurs. En 1852, Flaubert écrit à Louise Colet : « Voilà ce que tous les socialistes du monde n'ont pas voulu voir, avec leur éternelle prédication matérialiste. Ils ont nié la Douleur, ils ont blasphémé les trois quarts de la poésie moderne, le sang du Christ qui se remue en nous. - Rien ne l'extirpera, rien ne le tarira. Il ne s'agit pas de le dessécher, mais de lui faire des ruisseaux. Si le sentiment de l'insuffisance humaine, du néant de la vie venait à périr (ce qui serait la conséquence de leur hypothèse), nous serions plus bêtes que les oiseaux, qui au moins perchent sur les arbres $^{1}$.» Voilà qui est étonnant : la poésie moderne, du moins celle qui est digne de ce nom, serait donc un manifeste antisocialiste. Pourquoi ? Parce qu'elle n'est rien d'autre qu'un réceptacle pour le sang du Christ. Au nom du sang versé par le crucifié, de l'inanité de la vie et de l'insuffisance de tout ce qui est humain, la poésie moderne est un contre-discours face aux promesses messianiques vides et, pire encore, blasphématoires des socialistes de tous bords. Flaubert voit dans la pratique de la poésie moderne une forme d'imitatio Christi, qui, comme toute imitation, comporte un intense moment corporel : notre corps prend sur lui la douleur d'un autre corps. Ce n'est pas notre sang qui coule dans nos veines mais le sang du Christ qui fut sacrifié pour nous: "le sang du Christ qui se remue en nous». Seul le sang offert dans un sacrifice douloureux est éternel. Or, il existe à l'extérieur de nos corps un autre medium par lequel s'écoule le sang chrétien, un autre réceptacle : la poésie moderne ${ }^{2}$.

2 Mais que font ici les oiseaux qui " perchent sur les arbres ", plus intelligents que ceux qui se laissent berner par des promesses messianiques ? Ils suivent l'exemple du Christ, "perché sur l'arbre de la croix» (Ac 10,39). Dans une inspiration très hégélienne, Flaubert considère le monde et la Création comme le souvenir et la mise en scène de la Crucifixion. L'allusion implicite, cachée dans le jeu sémantique entre les expressions " perché sur les arbres » et " perché sur l'arbre de la croix », montre combien cette idée était familière à Flaubert. 
3 Hegel définit la Crucifixion comme une scène primitive de ce qu'il appela l'art romantique. Contrairement à l'art classique, l'art romantique ne peut pas se concevoir sans le christianisme. La scène primitive de l'art romantique, c'est l'Incarnation. Pour Hegel, l'Incarnation est un acte d'auto-renonciation souveraine, d'aliénation (kenosis) de Dieu qui se fait homme dans le Christ. Cette auto-humiliation radicale trouve son expression suprême dans la mort avilissante sur la croix. Selon Hegel, la particularité de l'art classique est constituée par son idéalité, cette beauté parfaite «qui élève les formes du monde visible au-dessus des conditions terrestres, qui efface les traces de l'existence mortelle et la remplace par une jeunesse florissante ${ }^{3}$. " Dans l'art classique, l'esprit se confond avec le corps. La beauté de l'âme se reflète dans la beauté physique. "La beauté grecque montre l'âme entièrement identifiée avec la forme corporelle; les divinités grecques révèlent leur individualité spirituelle dans leurs actions d'une manière toute extérieure, et leur félicité intérieure n'en est pas troublée ${ }^{4}$.» $\mathrm{Au}$ contraire, la scène primitive de l'art romantique est l'aliénation de Dieu qui s'est fait homme. La forme ne peut donc plus être le fondement ou la substance, comme elle l'était dans l'art classique. «Dans l'art romantique, l'union de l'âme avec l'absolu est un acte intérieur, qui, à la vérité, apparait dans l'extérieur, mais n'a pas celui-ci et sa forme réelle pour objet et pour but essentiel ${ }^{5}$.»

Dans l'art romantique, cet "acte intérieur " semble paradoxal dans la vérité objective des représentations sensibles : il apparaît sous la forme de l'inachevé, du laid ou, pour le dire plus clairement, dans l'inanité et l'anéantissement de l'apparence comme apparence ${ }^{6}$. Pour l'art romantique, le triomphe de la représentation se trouve dans sa propre impuissance, dans ce qui se soustrait à la représentabilité : «Parvenu au plus haut point de son développement, l'esprit se manifeste à lui-même dépouillé de toutes les formes extérieures de la matérialité. Invisible, il se saisit immédiatement par une pensée pure. C'est un son sans rien qui vibre à l'oreille, un vol au-dessus des airs, des accords qui se font entendre dans une sphère supérieure et dont les objets d'ici-bas ne peuvent recevoir qu'un faible écho ${ }^{7}$. »

5 Même si Erich Auerbach ne le dit pas expressément, ses essais sur le réalisme, qui, selon Hegel, doit être intégré à l'art romantique, se situent dans la tradition de l'esthétique hégélienne. Comme chez Hegel, l'approche d'Auerbach a le grand avantage d'écarter dès le départ la question de la représentation de la réalité. Auerbach préfère s'intéresser aux procédés littéraires qui produisent de la réalité, aux conditions de possibilité de la réalité ${ }^{8}$ pour parler comme Blumenberg, ou à l'« effet de réel ${ }^{9}$ » comme le dit Barthes. Comme Hegel, Auerbach considère essentiellement le réalisme comme la projection d'une scène paradoxale : l'incarnation du Verbe, l'incarnation de Dieu ${ }^{10}$. Sur ce plan purement formel, l'art romantique est un art chrétien. Autrement dit, la poésie conçue dans cette tradition a pour matrice cachée une réalité dont la conceptualisation a eu lieu à un moment historique précis, à savoir dans les textes des Evangiles. Flaubert ne connaît que trop bien cet héritage évangélique, il en est habité.

6 Selon la théorie d'Auerbach sur le réalisme, élaborée entre autres à partir de Madame Bovary ${ }^{11}$, celui-ci renverse la classique poétique des genres, en vigueur jusqu'au XVIII ${ }^{\mathrm{e}}$ siècle, qui associait rang éminent et style élevé. Le réalisme ne se moque pas des gens humbles et des choses simples de la vie quotidienne. Ils ne sont pas objets de comédie mais ils sont pris au sérieux, représentés avec sérieux, et ils peuvent même être tragiques. La description de leur existence provoque des sentiments qui n'étaient d'habitude attribués qu'au style élevé. 
7 Cette évolution stylistique ne commence pas au XIX siècle mais, comme l'écrit Auerbach dans son analyse de l'Antiquité latine tardive, dès le début du christianisme, avec le renversement de la hiérarchie rhétorique antique. L'irruption du christianisme dans le monde païen entraîna une rupture radicale avec toute la tradition rhétorique et littéraire. A la suite de Hegel, cette rupture a été interprétée d'un point de vue esthétique. S'appuyant sur Kojève, Alexandre Leupin parle d'une "coupure épistémologique chrétienne ", que Jean-Claude Milner reformule comme un théorème kojèvien : «Il n'y a jamais aucune synonymie entre une notion appartenant au système de pensée païen et une notion chrétienne, c'est-à-dire moderne. » Et Leupin déclare : "L'effort d'un Tertullien, par exemple, n'est autre qu'une vaste tentative d'homonymiser les noms de l'ancienne rhétorique pour leur faire dire autre chose - la réalité/vérité de l'Incarnation ${ }^{12}$. » Cette rupture est justifiée par un paradoxe inhérent à la révélation chrétienne : les Evangiles racontent en effet l'histoire inouïe d'un Dieu (annoncé comme un Messie) qui se fait homme. Boèce, qui écrit vers 512 deux traités intitulés Contre Eutychès et Nestorius, explique ainsi le paradoxe logique de l'Incarnation : "Quelle grande et nouvelle chose - unique et non-répétable en aucune époque - que la nature de celui qui est seul Dieu se joigne à la nature humaine (entièrement différente, elle, de Dieu), et forme ainsi une seule personne par la conjonction (copulatione) de natures différentes ${ }^{13}$ !». Mais ce n'est pas tout. Pour comble, ce dieu ne nait pas dans un palais mais dans une étable, parmi les plus pauvres. C'est la pauvreté qui est la signature décisive de cet avènement, de cette Incarnation. Dès le départ, le christianisme s'est caractérisé par l'insistance sur ce qui est humble et méprisé dans une société extrêmement stratifiée et hiérarchisée. En ce sens, le réalisme des pauvres est à l'origine une invention chrétienne.

8 Mais la question décisive sur le plan structurel se trouve ailleurs. La mort sur la croix est la matrice de la représentation du dieu fait homme sur cette Terre ${ }^{14}$. Lui qui est le Créateur de toute chose, met un terme à son existence en se laissant condamner, frapper, honnir, moquer et couvrir de crachats, dans la plus abjecte de toutes les morts ${ }^{15}$. Le triomphe de la représentation de cette croix est un triomphe dans lequel s'effondre l'économie antique de différenciation des styles, la distinction entre l'humble et le sublime. Elle révèle un moment de l'irreprésentable, qui résume à un niveau inouï d'abstraction théologique ce qui avait été exprimé dans la simple prophétie d'un Messie.

9 L'épanouissement de l'art romantique dans le système hégélien n'est rien d'autre que l'explication de cet événement originel. Dans sa lecture post-théologique de l'approche réformatrice de Luther, la beauté de ce qui est représenté apparaît de façon sublime parce qu'elle se cache dans l'inanité du monde: «Christus humilis, vos superbi». A ce propos, Auerbach rapproche trois phénomènes: 1) l'humilité du style de la Bible sermo humilis - qui fut écrite non par des rhéteurs érudits mais, aux yeux du public cultivé, sans doute par des sectaires fanatiques et à demi-incultes, dans un style bizarrement barbare et vulgaire et où abondaient d'absurdes superstitions au caractère infantile ; 2) l'humilité de l'objet qui correspond au style et 3 / l'inculture de ceux à l'oreille ou plutôt au cœur desquels devait parvenir la Bonne nouvelle.

10 Pour donner un exemple de l'humilitas de l'Incarnation, Auerbach cite la lettre de Paul aux Philippiens où est évoquée la " formam servi » que Jésus a choisie pour vivre la mort d'un esclave, sur la croix (Phil II, 5-9 ${ }^{16}$. Cette humiliation dans l'humain (kenosis), Luther l'a traduit par « inanité »; Hegel quant à lui traduit le concept grec de kenosis 
par «aliénation ». Selon la conceptualité hégélienne, le réalisme d'Auerbach, héritier du sermo humilis chrétien, est un style kénotique d'aliénation extrême. Quel est le point commun entre une poétique kénotique caractérisant l'art romantique ou moderne selon Hegel, et le réalisme de Flaubert, le « réalisme cryptique » (Pierre-Marc der Biasi) de l'Education sentimentale et le "monde du kitsch » (Marc Föcking) propre à Emma Bovary? Que signifie la définition de la poésie moderne donnée par Flaubert dans sa lettre à Louise Colet ? Gerald Wildgruber situait déjà la pratique scripturale de Flaubert, son travail sur l'idéal stylistique de l'impersonnalité, dans la tradition d'une imitatio Christi spécifique dont les derniers grands modèles remontent à la spiritualité française du XVII siècle. On le sait, Flaubert était profondément anticlérical. La contribution d'Agnès Bouvier, "Jéhovah égale Moloch », donne un exemple de la furor littéraire qui s'empare de Flaubert dans sa description des cultes idolâtres de Carthage, quand il dénonce non seulement les rites juifs mais aussi les pratiques de l'Eglise catholique romaine, la vénération de Marie, des saints ou du pape. Et bien que nous sachions aussi que l'anticléricalisme n'exclue pas forcément la religiosité, nous ne tenterons pas comme l'a fait Thibaudet de montrer que Flaubert était sur le point de se convertir.

Mais à la suite du curieux revirement religieux de la République Française laïque qui fait de ses professeurs de littérature des apôtres et des auteurs canoniques de la Grande nation des pères de l'Eglise, quelque chose a été refoulé dans l'écriture flaubertienne. Malgré les espoirs des professeurs républicains, l'écriture de Flaubert n'est pas neutre vis-à-vis de la matrice chrétienne que Hegel discerne dans l'art moderne, mais elle ne se situe pas non plus en opposition, voire au-delà d'elle. Flaubert élabore une poétique de la kénose, de l'aliénation et de l'humiliation, afin de mettre en scène le scandale de l'Evangile dans une ambivalence nouvelle et renforcée ${ }^{17}$. Le réalisme de Flaubert est la pratique d'une kénose poétique, dont les articles publiés ici tentent de cerner comment son principe est constitutif de réalité.

Flaubert, tel qu'il apparaît dans de nombreuses contributions rassemblées ici, est un autre Flaubert. Ce Flaubert différent est dû au travail collaboratif du CNRS et de l'Université de Munich sur «Flaubert: Religions, Antiquité, Création ", subventionné par l'ANR (Agence nationale de la recherche) et la DFG (Deutsche Forschungsgemeinschaft). Ce groupe de chercheurs a mis au jour les relations existant entre la mythographie européenne et l'œuvre de Flaubert qui n'étaient qu'esquissées jusqu'alors. Ils ont ainsi révélé une dimension profonde et méconnue de son œuvre, qui ne peut être simplement subsumée sous le concept de sécularisation.

\section{Sélection des articles traduits dans ce dossier}

Bouvier, Agnès : "Jéhovah égale Moloch: une lecture "antireligieuse" de Salammbô ", Romantisme, 136, 2007, p. 109-120.

Dufour, Philippe : "Salammbô, un tigre de marbre », dans : Gustave Flaubert 5, éd. par G. Séginger, Paris / Caen, 2005, p. 193-214.

De Biasi, Pierre-Marc : “"Qu'est-ce que cela veut dire, la réalité?” Le cryptage du réel dans L'Éducation sentimentale », dans : Vinken, B. / Fröhlicher, P. (éd.) : Le Flaubert réel, Tübingen: Niemeyer, 2009, p. 61-78.

Föcking, Marc: "Arkadien - eine Kitschwelt? Schwundstufen des Arkadischen in Flauberts Madame Bovary", dans: Friedlein, R. / Poppenberg, G. / Volmer, A. (éd.) : Arkadien in den romanischen Literaturen, Heidelberg: Winter, 2008, p. 483-492. 
Koppenfels, Martin von: "Flauberts Hand. Strategien der Selbstimmunisierung », Poetica, 34, 2002, p. 171-191.

Matthey, Cécile : «Itinéraire d'une croyance: Hérodias ", dans : Neefs, J. (éd.) : Savoirs en récit, t. II : Éclats de savoirs: Balzac, Nerval, Flaubert, Verne, les Goncourt, Presses Universitaires de Vincennes, 2010, p. 85-92.

Triaire, Sylvie : "Le défilé des dieux, de l'idole primitive à Loulou », dans : Herschberg Pierrot, A. (éd.) : Flaubert: les pouvoirs du mythe, avant-propos de B. Vinken et de P.-M. de Biasi, Publication Fractal, ANR-DFG [Actes du séminaire Flaubert 2008-2009, ITEM-Paris 8-ENS], Paris : Éditions Archives Contemporaines, sous presse.

2 Vinken, Barbara : "Sermo humilis: Realismus und Christentum » und " Notre-Dame de Rouen: Flauberts Kathedrale », dans : Flaubert. Durchkreuzte Moderne, Francfort-sur-leMain : Fischer, 2009, p. 350-374.

Wildgruber, Gerald : « Kunst - Religion - Wissenschaft. Zur Konstellation dreier Terme im Spätwerk Flauberts», Hofmannsthal Jahrbuch,7, 1999, p. 307-344.

\section{BIBLIOGRAPHIE}

Auerbach, Erich (1946) : Mimesis. Dargestellte Wirklichkeit in der abendländischen Literatur, Berne : Francke.

Auerbach, Erich (1958) : Literatursprache und Publikum in der lateinischen Spätantike und im Mittelalter, Berne : Francke.

Blumenberg, Hans (1964) : « Wirklichkeitsbegriff und Möglichkeit des Romans », in : Jauss, H. R. (éd.) : Nachahmung und Illusion, Munich : Fink (Poetik und Hermeneutik 1).

Culler, Jonathan (1975) : Structuralist Poetics, Londres / Ithaca : Routledge and Kegan Paul / Cornell University Press.

Flaubert, Gustave (1980) : Correspondance, t. II, éd. par J. Bruneau, Paris : Gallimard.

Hegel, Georg Friedrich Wilhelm (1997) : Esthétique, trad. par Ch. Bénard rev. et compl. par B. Timmermans et P. Zaccaria, Paris : Le Livre de poche.

Le Brun, Jean (2002) : Du pur amour. De Platon à Lacan, Paris : Seuil.

Leupin, Alexandre (1993) : Fiction et incarnation. Littérature et théologie au Moyen Age, Paris : Flammarion.

\section{NOTES}

1. Lettre à Louise Colet, 4 septembre 1852, Flaubert (1980), p. 151 sq.

2. Cela correspond à la thèse de Jean Le Brun (2002), selon laquelle le discours du pur amour est passé de la théologie à la littérature. Il l'illustre en prenant l'exemple du bref papal Cum alias, en 1699, dans lequel Innocent XII condamna l'Explication des maximes des saints de Fénelon, parue 
deux ans plus tôt. Malgré cette condamnation, la théologie du pur amour n'aurait pas pris fin ; la conception du pur amour serait passée dans un autre medium, le discours littéraire et psychanalytique. Flaubert revendique ici tout naturellement quelque chose de semblable. Ce ne sont plus l'Eglise et les écrits théologiques, mais la poésie moderne - notre corps à tous - qui est le lieu du souvenir, de la compréhension et de la préparation du sacrifice amoureux.

3. Hegel (1997), p. 656 sq.

4. Hegel (1997), p. 660.

5. Ibid.

6. Voir la contribution de Philippe Dufour «Salammbô, un tigre de marbre » consacrée à la représentation perturbante de la violence dans Salammbô. Dufour voit dans l'omission totale de commentaires narratifs ironiques un genre de pathos au deuxième degré, qui indique une affectation esthétique par l'objet.

7. Hegel (1997), p. 657.

8. Blumenberg (1964), p. 9-27.

9. Culler (1975), p. 38 sq. et p. 193.

10. Auerbach (1958), p. 53.

11. Auerbach (1946), p. 433.

12. Leupin (1993), p. 10.

13. Leupin (1993), p. 11.

14. Sur la représentation du Christ dans La tentation de saint Antoine et dans Trois contes de Flaubert avec en arrière-plan l'histoire des religions vue par Max Müller et Ernest Renan, voir la contribution de Sylvie Triaire «Le défilé des dieux, de l'idole primitive à Loulou ».

15. Auerbach (1958), p. 42, cite saint Augustin, Enarrationes in Psalmos 96.4 (CCSL 39, 1356-57; PL 37, 1239), que je paraphrase.

16. Traduction de Luther: «Ayez en vous les sentiments qui étaient en Jésus Christ lequel, existant en forme de Dieu, n'a point regardé comme une proie à arracher d'être égal avec Dieu mais s'est dépouillé lui-même, en prenant une forme de serviteur, en devenant semblable aux hommes; et ayant paru comme un simple homme il s'est humilié lui-même, se rendant obéissant jusqu'à la mort, même jusqu'à la mort de la croix. "

17. Voir B. Vinken, «Trois contes. Sermo humilis und Christentum » ainsi que Cécile Matthey, qui étudie le mystère de l'Incarnation sous l'angle de l'hospitalité, ce qui met au centre l'idée de la propagation et du partage, autrement dit l'idée de l'universalisme chrétien.

INDEX

Schlüsselwörter : Realismus, moderne Poesie, Christentum, Nichtigkeit, Hegel, Romantik

Mots-clés : réalisme, poésie moderne, christianisme, néant, Hegel, romantisme

\section{AUTEURS}

\section{BARBARA VINKEN}

Barbara Vinken est professeur de littérature et de philologie romane à l'université de Munich. 